\title{
O balanço de carbono da Amazônia brasileira
}

\author{
CARLOS A. NOBRE e ANTÓNIO D. NOBRE
}

\section{Introdução}

$\mathrm{N}$

BALANÇO global de carbono na atmosfera de nosso planeta, das cerca de 8 bilhões de toneladas de carbono emitidas anualmente na forma de dióxido de carbono $\left(\mathrm{CO}_{2}\right)$ pela queima de combustíveis fósseis e mudanças dos usos da terra, somente 3,2 bilhões permanecem na atmosfera, provocando o aumento do efeito estufa (aumento do aquecimento da superfície e da troposfera devido à absorção de radiação infravermelha termal por vários gases minoritários da atmosfera, principalmente o dióxido de carbono). O restante é reabsorvido pelos oceanos e pela biota terrestre. A Tabela 1 apresenta um resumo do balanço global de carbono para as décadas de 1980 e 1990, publicado no recente relatório do IPCC de 2001. Nota-se o grande papel da biota terrestre como o principal sumidouro do excesso de carbono atmosférico. Estima-se que esse sumidouro tenha sido responsável por retirar 1,9 gigatonelada de carbono por ano da atmosfera na década de 1980 e por um valor ainda maior na década de 1990. Para onde está indo esse carbono? Há evidências apontando que tanto as florestas temperadas como as florestas tropicais estão reassimilando parte desse excesso de $\mathrm{CO}_{2}$ atmosférico. Levando em conta que há um número 10 a 20 vezes maior de estudos do balanço de carbono para latitude médias do hemisfério norte em comparação com estudos do ciclo de carbono das florestas tropicais do planeta, o debate científico tem enfocado principalmente a questão de um gradiente leste-oeste do sumidouro, isto é, procura-se saber se o sumidouro encontra-se na América do Norte ou na Europa-Sibéria (veja, por exemplo, a discussão em Schimel, 2000).

À primeira vista, a Amazônia aparenta ser uma fonte de $\mathrm{CO}_{2}$ para a atmosfera, em função do desmatamento de entre 15 e 20 mil km² ao ano, somente na Amazônia brasileira (INPE, 2001). Entretanto, uma série de estudos recentes sobre o papel das florestas tropicais da Amazônia no ciclo de carbono abre a possibilidade de que também as florestas tropicais estejam desempenhando um papel relevante como sumidouros de $\mathrm{CO}_{2}$.

A questão adquire contornos práticos, além de ressaltar a necessidade de expandir o conhecimento científico sobre o comportamento dos grande ciclos naturais do planeta envolvendo carbono, água, nutrientes etc. No que concerne as ações para um possível controle das emissões globais, visando uma desejável estabilização da concentração dos gases-estufa na atmosfera, entender os mecanismos responsáveis pela absorção de $\mathrm{CO}_{2}$ atmosférico pelos oceanos e pela biota 
terrestre é essencial. Há várias hipóteses cientificamente bem fundamentadas dando conta de que o papel de amortecimento tanto dos oceanos como da atmosfera pode diminuir nas próximas décadas em virtude do aumento da concentração de $\mathrm{CO}_{2}$ atmosférico, da temperatura dos oceanos e, principalmente, da temperatura da biota terrestre (Prentice, 2001). Em outras palavras, conjectura-se que esses grandes sumidouros naturais poderiam reduzir sua magnitude no futuro, talvez em meados deste século, o que acarretaria, inevitavelmente, um aumento da taxa de crescimento das concentrações atmosféricas desse gás, a menos que se diminua significativamente as emissões globais. Em que pese o fato de que, atualmente, a grande maioria dos países com florestas tropicais em seus territórios não são obrigados por força de tratados internacionais (Convenção Quadro das Nações Unidas sobre Mudanças Climáticas e o resultante Protocolo de Quioto) a controlar ou reduzir emissões por serem países em desenvolvimento, determinar se a floresta tropical não-perturbada é sumidouro de carbono pode servir para demonstrar mais um importante serviço ambiental proporcionado pela floresta.

Tabela 1: Balanço global de $\mathrm{CO}_{2}$ (em $\mathrm{PgC} / \mathrm{ano}$ ) baseada em tendências intra-decadais de $\mathrm{CO}_{2}$ e $\mathrm{O}_{2}$ atmosféricos. Valores positivos indicam fluxos para a atmosfera (emissões); valores negativos representam retirada da atmosfera. Barras de erro representam incertezas $( \pm 1 \delta)$ e não a variabilidade interanual, que é substancialmente maior.

Década de 1980

Aumento na atmosfera

Emissões (combustível fóssil, produção de cimento)

Fluxo oceano-atmosfera

Fluxo superfície terrestre-atmosfera * particionado como

Mudança dos usos da terra

Sumidouro terrestre residual

$$
3,3 \pm 0,1
$$$$
5,4 \pm 0,3
$$$$
-1,09 \pm 0,6
$$$$
-0,2 \pm 0,7
$$$$
-1,7 \pm 0,5
$$$$
-1,4 \pm 0,7
$$

$3,2 \pm 0,1$

$6,3 \pm 0,4$

$1,7(0,6$ a 2,5$)$

n.d.

$-1,9(-3,8$ a 0,3$)$

n.d.

Fonte: tabela adaptada de Prentice, 2001, p. 190.

* O fluxo superfície terrestre-atmosfera representa o balanço de um termo positivo devido às mudanças dos usos da terra e um sumidouro terrestre residual. Os dois termos não podem ser separados com base nas medições atmosféricas atuais. Utilizando análises independentes para estimar o componente que se deve às mudanças dos usos da terra para a década de 1980, o sumidouro terrestre residual pode ser estimado para esta década. Entretanto, dados globais sobre as mudanças dos usos da terra para a década de 1990 não se encontram ainda disponíveis. 


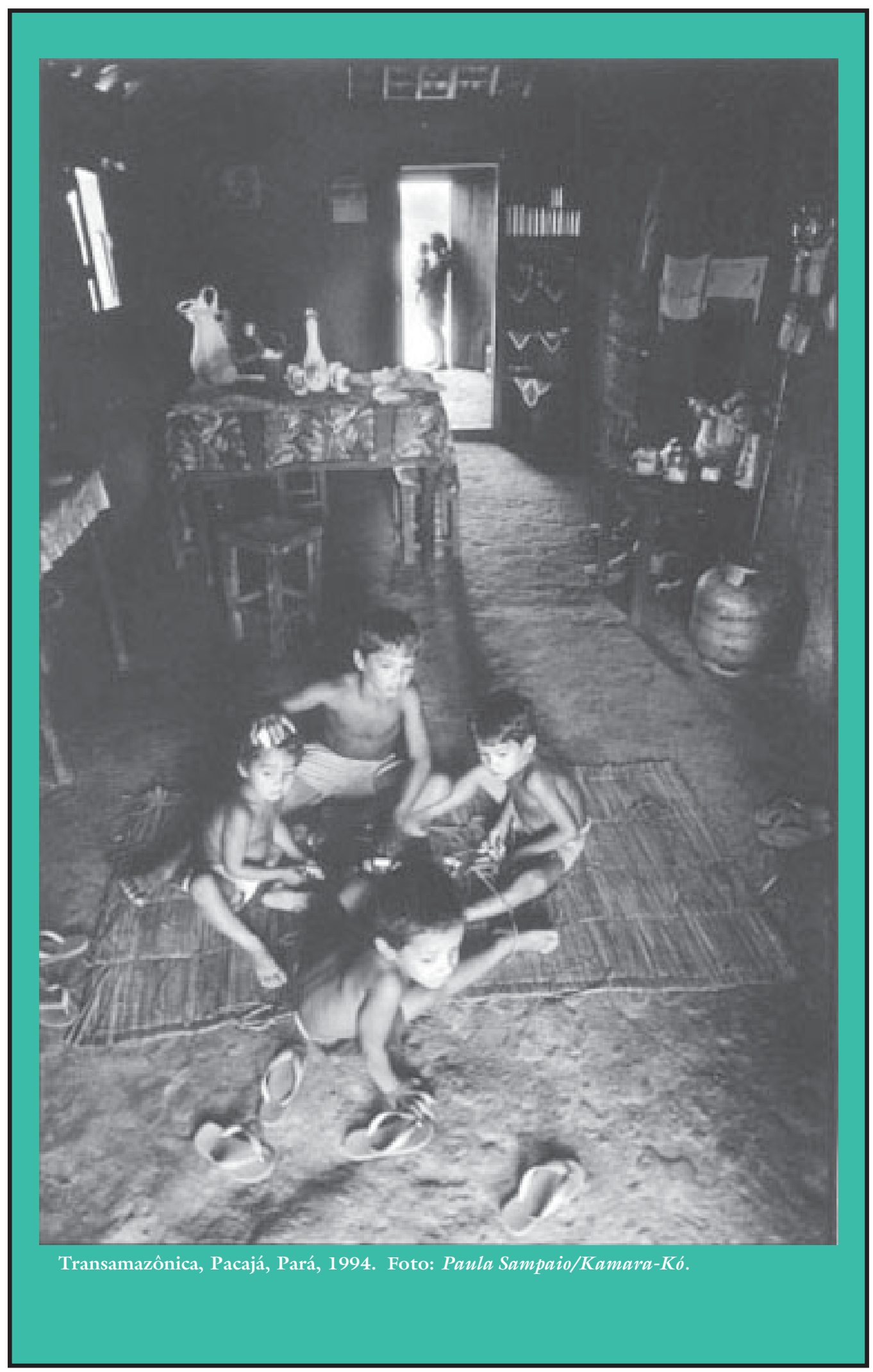

Estudos AvanÇAdos 16 (45), 2002 


\section{Uma breve revisão das observações}

A questão de ser a Amazônia fonte ou sumidouro de carbono para a atmosfera global permanece ainda em aberto. (Veja em Nobre, 2001 uma discussão mais aprofundada sobre o tema.) O desmatamento e a queima de biomassa foram responsáveis por emissões médias na década 1989-98 de 200 100 megatoneladas de carbono por ano (Houghton, 2000) na Amazônia brasileira, levando-se em conta a emissão por queima de biomassa do desmatamento, devido à decomposição relativamente rápida tanto da biomassa que resta após as queimadas como da matéria orgânica no solo, e pela retirada de madeira. Essas emissões correspondem a 10 a $15 \%$ das emissões globais devido a alterações dos usos da terra, estimadas em 1,7 $\pm 1,1$ gigatonelada de carbono por ano, como na tabela 1. Essa emissão de carbono para a atmosfera pode ser ainda maior, pois no cálculo acima não foram computadas as emissões resultantes das queimadas de florestas em pé, como, por exemplo, o mega-incêndio florestal de Roraima no primeiros meses de 1998. Normalmente, a floresta úmida é impenetrável ao fogo, pois a alta umidade torna a madeira pouco inflamável (Nepstad, 2000). Entretanto, a lenta degradação e fragmentação que a floresta vem sofrendo ao longo das últimas décadas, que se acelera com o aumento da extração seletiva de madeira por métodos não-sustentáveis, a torna suscetível ao fogo, pois a radiação solar pode penetrar mais facilmente, causando o secamento da madeira e aumentando sua flamabilidade. Essa situação é potencializada nas áreas da Amazônia sujeitas a secas prolongadas, como aquelas provocadas pelo fenômeno El Niño no norte e leste da região.

A conversão de toda a floresta Amazônica brasileira implica numa emissão total de cerca de 77 gigatoneladas de carbono para a atmosfera (Fearnside, 2000) e mais de 100 gigatoneladas de carbono para toda Amazônia. Por outro lado, medições realizadas pelos projetos científicos ABRACOS e LBA do fluxo líquido de $\mathrm{CO}_{2}$ para áreas de floresta não-perturbada (isto é, a diferença entre (a) o fluxo de $\mathrm{CO}_{2}$ da atmosfera para a floresta mediante o processo de assimilação de carbono através da fotossíntese durante o dia e (b) o fluxo de $\mathrm{CO}_{2}$ da floresta para atmosfera emitido pela respiração das plantas e devido à decomposição da matéria orgânica no chão da floresta e no solo), em cinco torres de medidas de fluxos no leste, centro e sudoeste da região, têm mostrado que a floresta aparentemente funciona como um sumidouro de parte do excesso de $\mathrm{CO}_{2}$ atmosférico, assimilando valores bastante expressivos, entre uma até nove toneladas de carbono por hectare por ano (Grace, 1996; Malhi, 1998; Malhi, 1999; Nobre, 2000; Araujo, 2002; Randow, 2002; Kruijt, 2002; Rocha, 2002; Goulden, 2002; Miller, 2002). Entretanto, a própria ampla faixa de valores encontrados nessas medidas pode indicar um alto grau de incerteza, além de variabilidade ecoclimática entre os diferentes pontos de medição. Estudos independentes baseados em inventários florestais (Phillips, 1998) e estimativas de fontes e sumidouros obtidos pelo método de inversão (conhecidas as concentrações de $\mathrm{CO}_{2}$ e $\mathrm{O}_{2}$ atmosféricos com grande precisão, calcula-se que a distribuição de fontes e sumidouros seja compatível 
com aquelas observações de concentrações) igualmente fornecem evidências observacionais do possível papel da Amazônia como sumidouro de $\mathrm{CO}_{2}$ atmosférico a taxas de 0 a 0,5 gigatonelada de carbono por ano. Entretanto, como ainda não se conhece os mecanismos que poderiam ser responsáveis pelo funcionamento da floresta como um sumidouro de modo a compensar as emissões devido às alterações dos usos da terra, ainda há incerteza para se concluir definitivamente sobre o balanço de carbono. Recentes cálculos do que poderia ser a emissão potencial de $\mathrm{CO}_{2}$ de rios, lagos, áreas alagadas na Amazônia central indicaram valores muito maiores do que se supunha anteriormente, com contribuições de até 1,2 $\pm 0,3$ tonelada de carbono por hectare por ano. A Figura l é uma tentativa de síntese preliminar do nosso conhecimento atual do ciclo de carbono para os florestais e aquáticos não-perturbados da Amazônia.

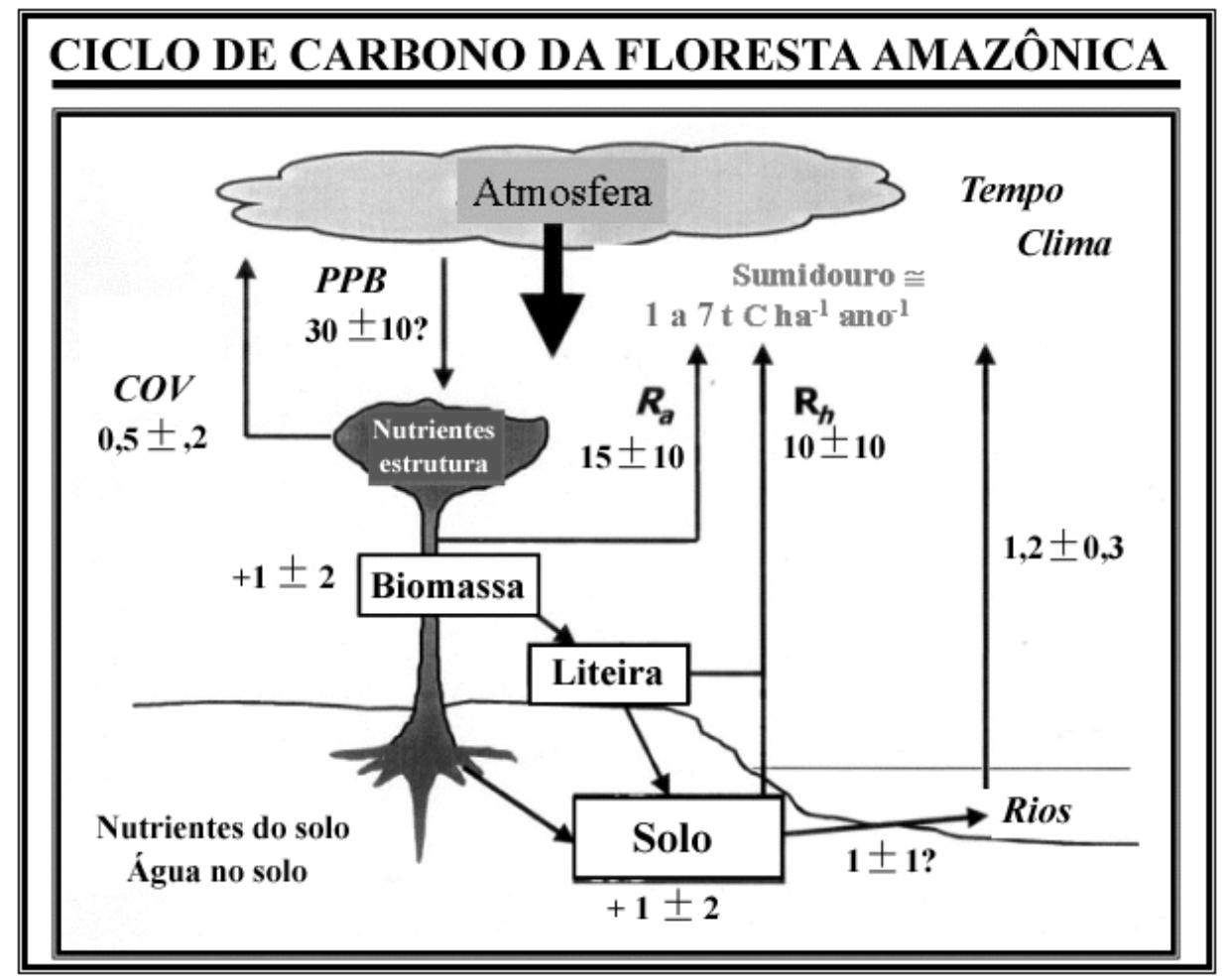

Síntese preliminar do ciclo de carbono da floresta tropical não-perturbada da Amazônia. Unidades: $\mathrm{t} C$ ha $^{-1}$ ano $^{-1}$. $P \boldsymbol{P B}=$ produtividade primária bruta; $\boldsymbol{R}_{a}$ = respiração autotrófica; $R_{b}=$ respiração heterotrófica; $C O V=$ compostos orgânicos voláteis. (Fonte: Projeto LBA, Consórcio Brasil-Europa [Alterra, INPA, IH, Edinburgh University] e CENA-USP e Washington University.)

Há questões metodológicas a serem respondidas. A mais comum delas indaga se as medidas nas torres de fluxos estão acuradamente capturando os fracos fluxos de $\mathrm{CO}_{2}$ à noite ou os fluxos para condições turbulentas logo no início do dia, quando a estável camada superficial noturna é quebrada e há um significati- 
vo fluxo para cima de $\mathrm{CO}_{2}$. Kruijt (2002) se debruçou sobre esta questão exaustivamente e seus resultados reiteram a existência do sumidouro. Sobre os mecanismos para explicar esse eventual sumidouro, uma possibilidade teórica seria simplesmente a resposta fisiológica do bioma a valores crescentes de $\mathrm{CO}_{2}$ atmosférico, o chamado efeito de "fertilização" de $\mathrm{CO}_{2}$. Uma outra possibilidade seria que a floresta encontra-se numa fase de recuperação após um período de alta mortalidade associada à alta variabilidade climática (por exemplo, maior freqüência de secas), como sugerido por Keller (1997). Cálculos do balanço de carbono da floresta Amazônica a partir de modelos (excluindo mudanças dos usos da terra) sugerem que a floresta não-perturbada é fonte de carbono durante anos de El Niño (mais secos no norte e leste da Amazônia) e sumidouro em anos de La Niña (chuvas mais abundantes) (Tian 1999). Entretanto, com o aquecimento global, esse sumidouro aparente pode saturar e tornar-se fonte, uma vez que um solo mais aquecido pode acelerar a respiração heterotrófica. Há sugestões de que mudanças climáticas podem levar a mudanças drásticas na vegetação da Amazônia, principalmente uma tendência à "savanização" (Cox, 2000). O desmatamento de grande escala pode resultar em tendências similares (Nobre, 1991).

O balanço de evidências observacionais até o presente não permite excluir a possibilidade de que as florestas tropicais da Amazônia estejam, de fato, funcionando como sumidouros de carbono. De qualquer modo, se esse resultado puder ser confirmado com novos e continuados estudos, ele representa um dado novo e alentador sobre o papel das florestas tropicais no balanço global de carbono. Esse sumidouro de carbono poderia, então, ser considerado como serviço ambiental adicional prestado pela floresta.

\section{Discussão e conclusões}

Afinal de contas, o bioma amazônico é sumidouro ou é fonte de carbono para a atmosfera? O projeto LBA, mediante uma variedade de experimentos, estudos observacionais e estudos de modelagem, está gerando um grande fluxo de novidades e surpresas sobre a Amazônia, que surgem como artigos em conceituadas revistas cientificas e pipocam na imprensa, muitas vezes em manchetes sensacionalistas. Uma avaliação superficial desses resultados, ainda parciais, pode passar uma imagem de aparente conflito dos achados em relação ao papel maior do bioma amazônico no ciclo do carbono. Contudo, uma análise mais cuidadosa e menos apaixonada indica um quadro muito menos contraditório, embora ainda incompleto, na complementaridade desses vários estudos. Medições contínuas em torres das trocas de longo prazo de $\mathrm{CO}_{2}$, entre a floresta e a atmosfera indicam, em sua maioria, um saldo na troca total de $\mathrm{CO}_{2}$ (emissão por respiração e seqüestro por fotossíntese), que varia de uma a nove toneladas de carbono por hectare por ano permanecendo na biomassa da floresta. A maioria dos estudos sistemáticos e de longo prazo, que monitoram o volume e crescimento da floresta em áreas contíguas às torres, encontram também aumento progressivo de biomassa de ano a ano, porém em valores bem menores do que aqueles indica- 
dos pelas medições nas torres de fluxos. Todavia, esses estudos de biomassa não conseguem encontrar o paradeiro de todo o carbono que estaria entrando e ficando na floresta. Num âmbito maior, embora mais incerto, resultados de observações em poucos locais das distribuições verticais e temporais de $\mathrm{CO}_{2}$ na camada limite da baixa atmosfera, utilizando aviões, indicam que, para a perspectiva da atmosfera, o bioma amazônico estaria em equilíbrio em termos de emissões e seqüestro, com uma soma cuja média se aproxima a zero. Ainda mais incertos, mas com sugestões semelhantes, estudos de distribuições de concentrações de $\mathrm{CO}_{2}$ em várias partes do globo, utilizando modelos de inversão, apontam para um sinal zero proveniente dos trópicos. Sinal zero nos trópicos significa também que nada do que é despejado na América do Sul, por exemplo, de $\mathrm{CO}_{2}$ oriundo da queima e decomposição de florestas derrubadas e de combustíveis fósseis aparece em outras partes do planeta. Esta possibilidade, por si só, validaria os resultados dos estudos de torres e biomassa que indicam as florestas de terra firme como sumidouros para o $\mathrm{CO}_{2}$ atmosférico. Ou seja, todo o $\mathrm{CO}_{2}$ produzido estaria sendo consumido aqui mesmo.

Não obstante, inferências físicas, biogeoquímicas e ecológicas podem indicar uma direção lógica para o comportamento desse bioma. Alguns estudos de modelagem global prevêem uma resposta de crescimento de florestas, como as da Amazônia, em uma atmosfera com maior concentração de $\mathrm{CO}_{2}$. Perfis que estudam concentrações de atmosferas passadas em microbolhas presas no gelo de geleiras ao redor do globo indicam que, por centenas de milhares de anos, $\mathrm{o} \mathrm{CO}_{2}$ atmosférico variou entre 180 e 290 ppm na atmosfera terrestre. Hoje essa concentração está em torno de 370 ppm! Além do efeito conhecido e comprovado do aumento de concentração deste que é o principal gás do efeito estufa no aquecimento do planeta, uma infinidade de estudos de enriquecimento de $\mathrm{CO}_{2}$ em casas de vegetação mostram que as plantas respondem ao $\mathrm{CO}_{2}$ como respondem a adubos minerais: crescem a taxas maiores, incorporando assim maior quantidade de carbono. Desse modo, não é absurdo supor que a própria floresta Amazônica esteja crescendo e, conseqüentemente, seqüestrando mais $\mathrm{CO}_{2}$ como resposta a esse grande experimento não-controlado de fertilização atmosférica com $\mathrm{CO}_{2}$. Como a concentração atmosférica de $\mathrm{CO}_{2}$ aumenta a taxas crescentes, reflexo do uso crescente de combustíveis fósseis e da acelerada destruição de florestas, é também de se supor que cada vez mais as florestas do planeta acelerem seu crescimento, até que encontrem limitações ecológicas e ecofisiológicas intrínsecas, sobre as quais conhecemos muito pouco ainda.

Como o bioma amazônico é um complexíssimo mosaico de ambientes em diferentes graus de alteração, cada um com comportamentos distintos em relação às trocas de carbono com a atmosfera, uma visão completa e crível do papel real de toda a região para a atmosfera somente poderá emergir quando os vários estudos integrarem seus resultados e quando os mecanismos físicos e biogeoquímicos forem melhor compreendidos em sua extensão e complexidade. 
Referências bibliográficas

ARAUJO, A.C.; A.D. Nobre; B. Kruijtz; A.D. Culd; Stefani; J. Elber; Dallarosa; C. Randow; A.O. Manzi; R. Valentini; J.H.C. Gash; P. Kabat. "Dual Tower Long-Term Study of Carbon Dioxide Fluxes for a Central Amazonian Rain Forest", Journal of Geophysical Research-LBA (2002). [Edição especial (no prelo).]

COX, P.M.; R.A. Betts; C.D. Jones; S.A. Spall; I.J. Totterdell. “Acceleration of Global Warming Due to Carbon-Cycle Feedbacks in a Coupled Climate Model. Nature 408: 184-187 (2000).

CIAIS, P.; P.P. Tans; J.W. White; M. Trolier; R. Francey; J. Berry; D. Randall; P. Sellers; J. Collatz; D.S. Schimel. "Partitioning of the Ocean and Land Uptake of $\mathrm{CO}_{2}$ from Delta ${ }^{13} \mathrm{C}$ Measurements from the NOAA/CMDL Global Air Sampling Network", Journal of Geophysical Research, 100(D3): 5051-5057 (1995).

FEARNSIDE, P. "O Potencial do Setor Florestal Brasileiro para a Mitigação do Efeito Estufa sob o Mecanismo de Desenvolvimento Limpo do Protocolo de Kyoto" em Moreira, A.G. e S. Schwartzman (eds.) As mudanças climáticas globais e os ecossistemas brasileiros (Brasília: UNB, 2002), pp. 59-74.

GOULDEN, M.L.; S.D. Miller; M.C. Menton; H.R. da Rocha; H.C. de Freitas. "Physiological Controls on Tropical Forest $\mathrm{CO}^{2}$ Exchange", submetido a Ecological Applications (2002).

GRACE, J.; J. Lloyd; J. Mcintyre; A. Miranda; P. Meir; H. Miranda; C.A. Nobre; J. Moncrieff; J. Massheder; Y. Malhi; I. Wright; J. Gash. "Carbon Dioxide Uptake by an Undisturbed Tropical Rain Forest in Sothwest Amazonia, 1992 to 1993”, Science, vol. 270, $\mathrm{n}^{\circ} 5237$, pp.778-780 (2002).

HOUGHTON, R.A.; D.L. Skole; C.A. Nobre; J.L. Hackler; K.T. Lawrence e W.H. Chomentowski. "Annual Fluxes of Carbon from Deforestation and Regrowth in the Brazilian Amazon”, Nature, 403: 301-304 (2000).

INSTITUTO NACIONAL DE PESQUISAS ESPACIAIS (INPE). Monitoramento da floresta Amazônica brasileira por satélite: 1998 - 2000 (São José dos Campos, Brasil: 2001) [http://www.inpe.br/Informacoes_Eventos/amazonia.htm.]

KELLER, M.; D.A. Clark; D.B. Clark; A.M. Weitz e E. Veldkamp. "If a Tree Falls in the Forest...", Science, 273:201 (1996).

KRUIJT, B.; J.A. Elbers; C. von Randow; A.C. Araujo; P.J. Oliveira; A. Culf; A.O. Manzi; A.D. Nobre e P. Kabat. "The Robustness in Eddy Correlation Fluxes for Amazon Rainforest Conditions", submetido a Ecological Applications (2002).

MALHI, Y.; A.D. Nobre; J. Grace; B. Kruijt; M.G.P. Pereira; A. Culf e S. Scott. "Carbon Dioxide Transfer over a Central Amazonian Rain Forest", Journal of Geophysical Research, D24: 31593-31612 (1998).

; D.D. Baldochi e P.G. Jarvis. "The Carbon Balance of Tropical, Temperate and Boreal Forests", Plant, Cell and Environment, 22: 715-740 (1999).

MILLER, S.D.; M.L. Goulden; M.C. Menton; H.R. da Rocha; H.C. de Freitas. "Annual $\mathrm{CO}^{2}$ Exchange by a Tropical Forest”, submetido a Ecological Applications (2002). 
NEPSTAD, D.C.; A. Verissimo; A. Alencar; C.A. Nobre; E. Lima; P. Lefebvre; P. Schlesinger; C. Potter; P. Moutinho; E. Mendonza; M. Cochrane e V. Brooks. "Large Scale Improverishment of Amazonian Forests by Logging and Fire", Nature, 398 (6727): 505-508 (1999).

NOBRE, A.; Y. Malhi; A.C. Araujo; A.D. Culf; A.D. Dolman; J. Elbers; B. Kruijt; C. Randow; A.O. Manzi; J. Grace e P. Kabat. "Multiyear Comparative Analysis of NEP and Environmental Factors for Manaus Rainforest: 'La Niña' Influence on $\mathrm{CO}_{2}$ Uptake”, First LBA Science Conference (Belém: 25-30 de junho de 2000). [http:// sauva.cptec.inpe.br/posters/.]

; P. Sellers e J. Shukla. "Regional Climate Change and Amazonian Deforestation Model", Journal of Climate, 4(10): 957-988 (1991).

“Amazônia: Fonte ou Sumidouro de Carbono?” em Ministério do Meio Ambiente do Brasil. Causas e dinâmica do desmatamento na Amazônia (Brasília: Ministério do Meio Ambiente, 2001), 436 p.

PHILLIPS, O.L.; Y. Malhi; N. Higuchi; W.F. Laurance; R.M. Núñez; D.J.D. Váxquez; L.V. Laurance; S.G. Ferreira; M. Stern; S. Brown e J. Grace. "Changes in the Carbon Balance of Tropical Forests: Evidence from Long-Term Plots”, Science, 282, 439-442 (1998).

PRENTICE, I. C.; G. D. Farquar; M. J. R. Fasham; M. L. Goulden; M. Heiman; V. J. Jaramillo; H. S. Kheshgi; C. Le Quéré; R. J. Scholes; D. W. R. Wallace et alii. "The Carbon Cycle and the Atmospheric Carbon Dioxide" em Houghton, J.T.; Y. Ding; D.J. Griggs; M. Noguer; P.J. van der Linden; X. Dai; K. Maskell e C. A. Johnson (eds.) Climate change 2001: the scientific basis, contribution of working group I to the third assessment report of the intergovernamental panel on climate change (Cambridge: Cambridge University Press, 2001), 881 p.

RANDOW, C.; D.A. von Leonardo; S. S Prasad; D. Gannabathula; Antonio O. Manzi; Paulo R. A. Arlino e B. Kruijt, 2001. "Scale Variability of Surface Fluxes of Energy and Carbon over a Tropical Rain Forest in South West AMAZONIA: I, Diurnal Conditions", aceito pelo Journal of Geophysical Research (2001) [Edição especial da LBA.]

RAYNER, P.J., I.G. Enting; R.J. Francey e R. Langefelds. "Reconstructing the Recent Carbon Cycle from Atmospheric $\mathrm{CO}_{2}, \mathrm{D}^{13} \mathrm{C}$ and $\mathrm{O}_{2} / \mathrm{N}_{2}$ Observations", Tellus, 51B, 213 (1999).

RICHEY, J.E.; J.M. Melack; A.K. Aufdenkampe; V.M. Balelster e L. Hess. “Outgassing from Amazonian Floodwaters as a Large Tropical Source of Atmospheric Carbon Dioxide", Nature, 416: (6881): 617-620 (2002).

ROCHA, H.R. da; M.L. Goulden; S.D. Miller; M.C. Menton; L.D.V. Oliveira Pinto; H.C. de Freitas e A.M.S. Figueira. "Seasonality of Water and Heat Fluxes over a Tropical Forest in Eastern Amazonia", submetido a Ecological Applications (2002).

SCHIMEL, D. J. Melillo; H. Tian; A. D. McGuire; D. Kicklighter; T. Kittel; N. Rosenbloom; S. Running; P. Thornton; D. Ojima; W. Parton; R. Kelly; M. Sykes; R. Neilson e B. Rizzo. "Contribution of Increasing $\mathrm{CO}^{2}$ and Climate to Carbon Storage by Ecosystems in the United States", Science, 287: 2004-2006 (2000).

TIAN, H.Q.; J. M. Melillo e D. W. Kicklighter. "Effect of Interanual Climate Variability on Carbon Storage in Amazonian Ecosystems", Nature, 396: 664-667 (1998). 
RESUMO - GLOBALMENTE, a biota terrestre é um sumidouro significativo de dióxido de carbono $\left(\mathrm{CO}_{2}\right)$ atmosférico. Estudos recentes do IPCC para a década de 1990 estimam a biota terrestre com sendo um sumidouro líquido de aproximadamente 1,4 gigatonelada de carbono por ano (assimilação líquida pela biota terrestre menos as emissões devidas às mudanças dos usos da terra). É provável que a maior parte desse suposto sumidouro aconteça nas florestas das latitudes médias e dos trópicos. Estudos do ciclo do carbono do Experimento LBA estão mostrando que as florestas não-perturbadas da Amazônia comportam-se com um forte sumidouro de carbono, com taxas na faixa de 1 a 7 toneladas por hectare por ano, ao passo que as áreas inundadas e os rios podem estar agindo como fonte de carbono de até 1,2 tonelada por hectare por ano. O desmatamento e a queima de biomassa representam uma emissão líquida de aproximadamente 0,2 gigatonelada de carbono por ano na Amazônia brasileira. Ainda que se leve em conta as grandes incertezas existentes sobre essas medidas, o balanço das evidências observacionais aponta para a possibilidade de que as florestas tropicais da América do Sul estejam funcionando como sumidouros de carbono da atmosfera.

ABSTRACT - GLOBALLY, the terrestrial biota acts as a significant carbon sink for atmospheric carbon dioxide $\left(\mathrm{CO}_{2}\right)$. The most recent estimate from IPCC for the 1990's puts the terrestrial biota at a net sink of 1.4 gigaton of carbon per year (net carbon uptake by the biota minus emissions from land use changes). It is likely that most of this presumed sink takes place in mid-latitude and tropical forests. Carbon cycle studies in the LBA Experiment indicate that the undisturbed forest of Amazonia may be a strong sink of carbon, at rates from 1 to 7 tons per hectare per year, whereas the wetlands may act as a source of carbon into the atmosphere of up to 1.2 ton per hectare per year. Deforestation and biomass burning in Brazilian Amazonia alone account for a net carbon dioxide emission of about 0,2 gigaton of carbon per year. Notwithstanding the still large uncertainties of these estimates, the balance of observational evidence points to the possibility that the tropical forests of South America function as a sink of carbon from the atmosphere.

Carlos A. Nobre é pesquisador do Centro de Previsão de Tempo e Estudos Climáticos (CPTEC--NPE), Cachoeira Paulista, SP. nobre@cptec.inpe.br .

Antônio D . Nobre é pesquisador do Instituto Nacional de Pesquisas da Amazônia (INPA), Manaus, AM. anobre27@yahoo.com . 